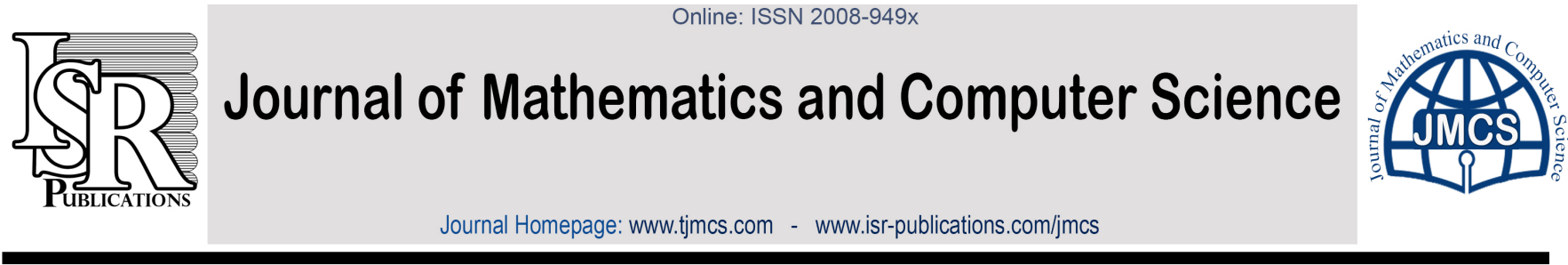

\title{
Convergence analysis of the numerical method for a singularly perturbed periodical boundary value problem
}

\author{
Musa Cakira , Ilhame Amiralib,*, Mustafa Kuduc,*, Gabil M. Amiraliyev ${ }^{c}$ \\ ${ }^{a}$ Department of Mathematics, Faculty of Science, Yuzuncu Yil University, 65080, Van, Turkey. \\ ${ }^{b}$ Department of Mathematics, Faculty of Art and Sciences, Duzce University, 81620, Duzce, Turkey. \\ ${ }^{c}$ Department of Mathematics, Faculty of Art and Sciences, Erzincan University, 24000, Erzincan, Turkey.
}

\begin{abstract}
This work deals with the singularly perturbed periodical boundary value problem for a quasilinear second-order differential equation. The numerical method is constructed on piecewise uniform Shishkin type mesh, which gives first-order uniform convergence in the discrete maximum norm. Numerical results supporting the theory are presented. (c)2016 All rights reserved.
\end{abstract}

Keywords: Singular perturbation, periodical problem, fitted difference method, uniformly convergent, boundary layer.

2010 MSC: 65L12, 65L70, 34B10, 34D15.

\section{Introduction}

In this paper, we consider the non-linear second order singularly perturbed periodical boundary value problem (BVP)

$$
\begin{gathered}
L u \equiv \varepsilon^{2} u^{\prime \prime}+\varepsilon a(x) u^{\prime}-f(x, u)=0,0<x<l, \\
u(0)-u(l)=0, \\
L_{0} u \equiv \varepsilon\left(u^{\prime}(l)-u^{\prime}(0)\right)=A,
\end{gathered}
$$

where $0<\varepsilon<<1$ is the perturbation parameter and $A$ is a given constant. We assume that the functions $a(x) \geq 0$ and $f(x, u)$ are sufficiently smooth on $[0, l]$ and $[0, l] \times \mathbb{R}$, respectively, to be specified and besides $a(0)=a(l)$ and $f(0, u(0))=f(l, u(l))$, and furthermore

$$
0<\beta \leq \frac{\partial f}{\partial u} \leq \beta^{*}<\infty
$$

The solution $u$ of (1.1)-1.3 has in general a boundary layer near $x=0$ and $x=l$ (see Section 2).

\footnotetext{
${ }^{*}$ Corresponding author

Email addresses: cakirmusa@hotmail.com (Musa Cakir), ailhame@gmail.com (Ilhame Amirali), muskud28@yahoo.com (Mustafa Kudu), gabilamirali@yahoo.com (Gabil M. Amiraliyev)
} 
Numerical treatment of singular perturbation problems has received a great deal of attention in the past. This type of problems arise in various fields of applied mathematics, mechanics and physics, see [5, 6, 11, 12, 15] and also references therein.

It is a well known fact that the solution of singularly perturbed boundary value problems exhibit a multiscale character. That is, there is a thin layer(s) where the solution varies rapidly, while away from the layer the solution behaves regularly and varies slowly. So, the standard numerical methods for solving such problems are unstable and fail to give accurate results when the perturbation parameter $\varepsilon$ is small. Therefore, it is important to develop suitable numerical methods for solving these problems, whose accuracy does not depend on the value of parameter $\varepsilon$, that is methods that are convergence $\varepsilon$-uniformly. One of techniques used to derive such methods consists of using fitted difference schemes on the special condensing grids (see [6, 7, 9, 10, 17] and also references cited in them). The difference schemes for singularly perturbed periodical problems with first order reduced equation have been handled by other techniques examined in the works of Pechenkina [13], Lin and Jiang [8], Xin [3] and the references therein. Extra inquiry regarding to numerical solution of periodical and other type problems can be also found in survey paper of Ramos [14.

Our goal in this paper is to give an exponentially fitted difference scheme on a special piecewise uniform grid (Shishkin grid) for the numerical solution of (1.1)-(1.3). We construct a difference scheme that is based on the method of integral identities by using exponential basis functions and interpolating quadrature rules with the weight and remainder terms in integral form, see [1, 2, 4. This method of approximation has the advantage that the schemes can also be effective in the case where the original problem considered under certain singularities. In the Section 2 we give useful properties of the exact solution of (1.1)-(1.3) that are needed in later sections. In Section 3 , we construct exponentially finite fitted difference scheme using a piecewise uniform mesh, which is fitted to the boundary layers and give the error analysis for the approximate solution. Uniform convergence is proved in the discrete maximum norm. Some numerical results, which are in agreement with the theoretical results have presented in the Section 4. The approach to the construction of the discrete problem and the error analysis for the approximate solution are similar to those in the works of Amiraliyev and Mamedov [2] and Amiraliyev and Duru [1].

Throughout the paper, $C$ will denote a generic positive constant independent of $\varepsilon$ and the mesh parameter.

\section{Properties of the exact solution}

Here we give useful asymptotic estimates of the exact solution of the problem (1.1)-(1.3) that are needed in later sections.

Lemma 2.1. Let $a, f \in C^{1}[0, l]$. Then for the solution $u(x)$ of the problem (1.1)-1.3 the following estimates hold:

$$
\|u\| \leq \beta^{-1}\|F\|+\bar{\beta}|A|,
$$

where

$$
\begin{aligned}
\|u\| & =\max _{[0, l]}|u(x)|, \quad \bar{\beta}=c_{0} \operatorname{coth}\left(c_{0} l / 4\right), \quad c_{0}=a^{*}+\sqrt{\left(a^{*}\right)^{2}+4 \beta}, \\
a^{*} & =\max _{[0, l]} a(x), \quad F(x)=f(x, 0)
\end{aligned}
$$

and

$$
\left|u^{\prime}(x)\right| \leq C\left\{1+\varepsilon^{-1}\left(e^{\frac{-\mu_{1} x}{\varepsilon}}+e^{\frac{-\mu_{2}(l-x)}{\varepsilon}}\right)\right\}, \quad 0 \leq x \leq l
$$

with

$$
\mu_{1}=0.5\left(\sqrt{a^{2}(0)+4 \beta}+a(0)\right), \quad \mu_{2}=0.5\left(\sqrt{a^{2}(l)+4 \beta}-a(l)\right),
$$

providing that $\frac{\partial f}{\partial x}(x, u)$ is bounded for $x \in[0, l]$ and $|u| \leq C$. 
Proof. We rewrite $(1.1)$ in the form

$$
\begin{aligned}
L_{*} u & \equiv \varepsilon^{2} u^{\prime \prime}+\varepsilon a(x) u^{\prime}-b(x) u \\
& =F(x), 0<x<l
\end{aligned}
$$

with $b(x)=\frac{\partial f}{\partial u}(x, \xi u), 0<\xi<1$ and use the Maximum Principle: Let $L_{*}$ and $L_{0}$ be the differential operators in (2.3), 1.2$)-(1.3)$ and $v \in C^{2}[0, l]$. If $v(0)=v(l), L_{0} v \geq 0$, and $L_{*} v \leq 0$ for all $0<x<l$, then $v(x) \geq 0$ for all $0 \leq x \leq l$. The further analysis is almost identical to that in the work of Amiraliyev and Duru [1].

\section{Discretization, layer-adapted mesh and convergence}

Let $\omega_{N}$ be any nonuniform mesh on $[0, l]$ :

$$
\omega_{N}=\left\{0<x_{1}<\ldots<x_{N-1}<l, h_{i}=x_{i}-x_{i-1}\right\}
$$

and $\bar{\omega}_{N}=\omega_{N} \cup\left\{x_{0}=0, x_{N}=l\right\}$. To simplify the notation we set $v_{i}=v\left(x_{i}\right)$ for any function $v(x)$, while $y_{i}$ denotes an approximation of $u(x)$ at $x_{i}$. For any mesh function $\left\{v_{i}\right\}$ defined on $\bar{\omega}_{N}$ we use

$$
\begin{aligned}
v_{\bar{x}, i} & =\frac{v_{i}-v_{i-1}}{h_{i}}, \quad v_{x, i}=\frac{v_{i+1}-v_{i}}{h_{i+1}}, \\
v_{\grave{x}, i} & =\frac{v_{\bar{x}, i}+v_{x, i}}{2}, \quad v_{\hat{x}, i}=\frac{v_{i+1}-v_{i}}{\hbar}, \\
v_{\bar{x} \hat{x}, i} & =\frac{v_{x, i}-v_{\bar{x}, i}}{\hbar}, \quad \hbar_{i}=\frac{h_{i}+h_{i+1}}{2}, \\
\|v\|_{\infty} & \equiv\|v\|_{\infty, \bar{\omega}_{N}}:=\max _{0 \leq i \leq N}\left|v_{i}\right| .
\end{aligned}
$$

The approach to generating the difference method is through the integral identity

$$
\chi_{i}^{-1} \hbar_{i}^{-1} \int_{0}^{l} \operatorname{Lu} \varphi_{i}(x) d x=0, i=1,2, \ldots, N-1
$$

with the exponential basis functions $\left\{\varphi_{i}(x)\right\}_{i=1}^{N-1}$ having the form

$$
\varphi_{i}(x)= \begin{cases}\varphi_{i}^{(1)}(x), & x_{i-1}<x<x_{i} \\ \varphi_{i}^{(2)}(x), & x_{i}<x<x_{i+1} \\ 0, & x \notin\left(x_{i-1}, x_{i+1}\right)\end{cases}
$$

where $\varphi_{i}^{(1)}(x)$ and $\varphi_{i}^{(2)}(x)$, respectively, are the solutions of the following problems:

$$
\begin{aligned}
\varepsilon \varphi^{\prime \prime}-a_{i} \varphi^{\prime} & =0, \quad x_{i-1}<x<x_{i} \\
\varphi\left(x_{i-1}\right) & =0, \quad \varphi\left(x_{i}\right)=1, \\
\varepsilon \varphi^{\prime \prime}-a_{i} \varphi^{\prime} & =0, \quad x_{i}<x<x_{i+1}, \\
\varphi\left(x_{i}\right) & =1, \quad \varphi\left(x_{i+1}\right)=0 .
\end{aligned}
$$

The functions $\varphi_{i}^{(1)}(x)$ and $\varphi_{i}^{(2)}(x)$ can be explicitly expressed as follows:

$$
\begin{aligned}
& \varphi_{i}^{(1)}(x)=\frac{e^{a_{i}\left(x-x_{i-1}\right) / \varepsilon}-1}{e^{a_{i} h_{i} / \varepsilon}-1}, \text { for } a_{i} \neq 0, \\
& \varphi_{i}^{(2)}(x)=\frac{1-e^{-a_{i}\left(x_{i+1}-x\right) / \varepsilon}}{1-e^{-a_{i} h_{i+1} / \varepsilon}}, \text { for } a_{i} \neq 0,
\end{aligned}
$$




$$
\begin{aligned}
& \varphi_{i}^{(1)}(x)=\frac{x-x_{i-1}}{h_{i}}, \quad \text { for } a_{i}=0, \\
& \varphi_{i}^{(2)}(x)=\frac{x_{i+1}-x}{h_{i+1}}, \quad \text { for } \quad a_{i}=0,
\end{aligned}
$$

and

$$
\begin{aligned}
\chi_{i} & =\hbar_{i}^{-1} \int_{x_{i-1}}^{x_{i+1}} \varphi_{i}(x) d x \\
& =\left\{\begin{array}{cc}
\hbar_{i}^{-1}\left(\frac{h_{i}}{1-e^{a_{i} h_{i} / \varepsilon}}+\frac{h_{i+1}}{1-e^{-a_{i} h_{i+1} / \varepsilon}}\right), & a_{i} \neq 0 \\
1, & a_{i}=0 .
\end{array}\right.
\end{aligned}
$$

Using interpolating quadrature rules with the weight and remainder terms in integral form on subinterval $\left[x_{i-1}, x_{i+1}\right]$, consistently with [1, 2, 4], we obtain the following relation:

$$
\begin{aligned}
\ell u_{i}+R_{i} & \equiv \varepsilon^{2} \theta_{i} u_{\bar{x} \hat{x}, i}+\varepsilon a_{i} u_{\dot{x}, i}-f\left(x_{i}, u_{i}\right)+R_{i} \\
& =0, \quad i=1,2, \ldots, N-1
\end{aligned}
$$

with

$$
\theta_{i}= \begin{cases}\frac{a_{i} \hbar_{i}}{2 \varepsilon}\left(\frac{h_{i+1}\left(e^{\frac{a_{i} h_{i}}{\varepsilon}}-1\right)+h_{i}\left(1-e^{-\frac{a_{i} h_{i+1}}{\varepsilon}}\right)}{h_{i+1}\left(e^{\frac{a_{i} h_{i}}{\varepsilon}}-1\right)-h_{i}\left(1-e^{-\frac{a_{i} h_{i+1}}{\varepsilon}}\right)}\right), & a_{i} \neq 0 \\ 1, & a_{i}=0\end{cases}
$$

and local truncation error

$$
\begin{aligned}
R_{i}= & -\ell u_{i} \\
= & \varepsilon \chi_{i}^{-1} \hbar_{i}^{-1} \int_{x_{i-1}}^{x_{i+1}}\left[a(x)-a\left(x_{i}\right)\right] u^{\prime} \varphi_{i}(x) d x-\chi_{i}^{-1} \hbar_{i}^{-1} \int_{x_{i-1}}^{x_{i+1}} d x \varphi_{i}(x) \\
& \times \int_{x_{i-1}}^{x_{i+1}} \frac{d}{d x} f(\xi, u(\xi)) K_{0, i}^{*}(x, \xi) d \xi
\end{aligned}
$$

where

$$
\begin{aligned}
K_{0, i}^{*}(x, \xi) & =T_{0}(x-\xi)-T_{0}\left(x_{i}-\xi\right), \\
1 & \leq i \leq N-1, \\
T_{0}(\lambda) & = \begin{cases}1, & \lambda \geq 0 \\
0, & \lambda<0 .\end{cases}
\end{aligned}
$$

To define an approximation for the boundary condition (1.3), we use the integral identity

$$
\int_{0}^{\ell} \operatorname{Lu} \varphi_{0}(x) d x=0
$$

with the exponential basis function $\varphi_{0}(x)$ having the form

$$
\varphi_{0}(x)= \begin{cases}\varphi_{0}^{(2)}(x), & x \in\left(x_{0}, x_{1}\right), \\ \varphi_{N}^{(1)}(x), & x \in\left(x_{N-1}, x_{N}\right), \\ 0, & \text { otherwise, }\end{cases}
$$

where $\varphi_{0}^{(2)}(x)$ and $\varphi_{N}^{(1)}(x)$, respectively, are the solutions of the following problems:

$$
\varepsilon \varphi_{0}^{\prime \prime}-a_{0} \varphi_{0}^{\prime}=0, \quad x_{0}<x<x_{1},
$$




$$
\begin{aligned}
\varphi_{0}\left(x_{0}\right)=1, & \varphi\left(x_{1}\right)=0, \\
\varepsilon \varphi_{N}^{\prime \prime}-a_{N} \varphi_{N}^{\prime} & =0, \quad x_{N-1}<x<x_{N}, \\
\varphi_{N}\left(x_{N-1}\right) & =0, \quad \varphi\left(x_{N}\right)=1 .
\end{aligned}
$$

Analogously, as above, we can write the following difference relation:

$$
\begin{aligned}
\ell_{0} u_{0} & \equiv \varepsilon^{2}\left(\theta_{0}^{(N)} u_{\bar{x}, N}-\theta_{0}^{(0)} u_{x, 0}\right)+\kappa_{0} f\left(0, u_{0}\right) \\
& =\varepsilon A-r
\end{aligned}
$$

with factor coefficients

$$
\begin{gathered}
\theta_{0}^{(0)}=\left\{\begin{array}{cc}
\frac{a_{0} h_{1} / \varepsilon}{1-e^{-a_{0} h_{1} / \varepsilon}} & a_{0} \neq 0 \\
1, & a_{0}=0
\end{array}\right. \\
\theta_{0}^{(N)}=\left\{\begin{array}{cc}
\frac{a_{N} h_{3} / \varepsilon}{e^{a_{N} h_{3} / \varepsilon}-1} & a_{N} \neq 0 \\
1, & a_{N}=0 \\
\kappa_{0}=\frac{h_{1}}{1-e^{-a_{0} h_{1} / \varepsilon}}-\frac{h_{3}}{e^{a_{N} h_{3} / \varepsilon}-1}
\end{array}\right.
\end{gathered}
$$

and local truncation error

$$
\begin{aligned}
r= & \varepsilon A-\ell_{0} u_{0} \\
= & \varepsilon \int_{x_{0}}^{x_{1}}\left[a_{0}-a(x)\right] u^{\prime}(x) \varphi_{0}^{(2)}(x) d x+\varepsilon \int_{x_{N-1}}^{x_{N}}\left[a_{N}-a(x)\right] u^{\prime}(x) \varphi_{N}^{(1)}(x) d x \\
& +\int_{x_{0}}^{x_{1}}\left[f(x, u)-f\left(0, u_{0}\right)\right] \varphi_{0}^{(2)}(x) d x+\int_{x_{N-1}}^{x_{N}}\left[f(x, u)-f\left(l, u_{N}\right)\right] \varphi_{N}^{(1)}(x) d x .
\end{aligned}
$$

Neglecting $R_{i}$ and $r$ in $(3.2)$ and (3.5), we have the following difference scheme for approximation (1.1)(1.3):

$$
\begin{gathered}
\ell y_{i} \equiv \varepsilon^{2} \theta_{i} y_{\bar{x} \hat{x}, i}+\varepsilon a_{i} y_{\grave{x}, i}-f\left(x_{i}, y_{i}\right)=0, \quad i=1,2, \ldots, N-1 \\
y_{0}-y_{N}=0, \\
\ell_{0} y_{0} \equiv \varepsilon^{2}\left(\theta_{0}^{(N)} y_{\bar{x}, N}-\theta_{0}^{(0)} y_{x, 0}\right)+\kappa_{0} f\left(0, y_{0}\right) \\
=\varepsilon A,
\end{gathered}
$$

where $\theta_{i}, \theta_{0}^{(0)}, \theta_{0}^{(N)}$ and $\kappa_{0}$ are defined by 3.3, , 3.6), 3.7) and (3.8), respectively.

We now give the mesh. The difference scheme (3.10)-(3.12) in order to be $\varepsilon$-uniform convergent, we will use the Shishkin mesh on $[0, l]$. For a divisible by 4 positive integer $N$, we divide each of the intervals $\left[0, \sigma_{1}\right]$ and $\left[l-\sigma_{2}, l\right]$ into $N / 4$ equidistant subintervals and also $\left[\sigma_{1}, l-\sigma_{2}\right]$ into $N / 2$ equidistant subintervals, where the transition points $\sigma_{1}$ and $\sigma_{2}$, which separate the fine and coarse portions of the mesh, are obtained by taking

$$
\sigma_{1}=\min \left\{\frac{l}{4}, \mu_{1}^{-1} \varepsilon \ln N\right\}, \quad \sigma_{2}=\min \left\{\frac{l}{4}, \mu_{2}^{-1} \varepsilon \ln N\right\},
$$

where $\mu_{1}$ and $\mu_{2}$ are given in Lemma 2.1. In practice, one usually has $\sigma_{i}<<l(i=1,2)$, so the mesh is fine on $\left[0, \sigma_{1}\right],\left[l-\sigma_{2}, l\right]$ and coarse on $\left[\sigma_{1}, l-\sigma_{2}\right]$. Hence, if denote by $h^{(1)}, h^{(2)}$ and $h^{(3)}$ the step-size in $\left[0, \sigma_{1}\right]$, $\left[\sigma_{1}, l-\sigma_{2}\right]$ and $\left[l-\sigma_{2}, l\right]$, respectively, we have 


$$
\begin{gathered}
h^{(1)}=\frac{4 \sigma_{1}}{N}, \quad h^{(2)}=\frac{2\left(l-\sigma_{2}-\sigma_{1}\right)}{N}, \quad h^{(3)}=\frac{4 \sigma_{2}}{N}, \\
\frac{\left(h^{(1)}+h^{(3)}\right)}{2}=\frac{2 l}{N}, \quad h^{(k)} \leq l N^{-1}, \quad k=1,3, \\
l N^{-1} \leq h^{(2)}<2 l N^{-1},
\end{gathered}
$$

so

$$
\bar{\omega}_{N}=\left\{\begin{array}{lc}
x_{i}=i h^{(1)}, & i=0,1, \ldots, \frac{N}{4} \\
x_{i}=\sigma_{1}+\left(i-\frac{N}{4}\right) h^{(2)}, & i=\frac{N}{4}+1, \ldots, \frac{3 N}{N} \\
x_{i}=l-\sigma_{2}+\left(i-\frac{3 N}{4}\right) h^{(3)}, & i=\frac{3 N}{4}+1, \ldots, N \\
h^{(1)}=\frac{4 \sigma_{1}}{N}, \quad h^{(2)}=\frac{2\left(l-\sigma_{2}-\sigma_{1}\right)}{N}, & h^{(3)}=\frac{4 \sigma_{2}}{N}
\end{array}\right.
$$

We now estimate the approximate error $z_{i}=y_{i}-u_{i}$, which satisfies

$$
\begin{gathered}
\varepsilon^{2} \theta_{i} z_{\bar{x} \hat{x}, i}+\varepsilon a_{i} z_{x}^{\circ}, i-\tilde{b}_{i} z_{i}=R_{i}, \quad i=1,2, \ldots, N-1, \\
z_{0}-z_{N}=0, \\
\varepsilon^{2}\left(\theta_{0}^{(N)} z_{\bar{x}, N}-\theta_{0}^{(0)} z_{x, 0}\right)+\bar{b}_{0} \kappa_{0} z_{0}=r,
\end{gathered}
$$

where

$$
\tilde{b}_{i}=\frac{\partial f}{\partial u}\left(x_{i}, \tilde{y}_{i}\right), \quad \bar{b}_{0}=\frac{\partial f}{\partial u}\left(0, \bar{y}_{0}\right),
$$

$\tilde{y}_{i}, \bar{y}_{0}$-intermediate points called for by the mean value theorem and the truncation errors $R_{i}$ and $r$ are defined by (3.4), (3.9), respectively. It is not difficult to observe that the discrete maximum principle is valid here and hereby

$$
\|z\|_{\infty, \bar{\omega}_{N}} \leq \beta^{-1}\|R\|_{\infty, \omega_{N}}+\left(\beta \kappa_{0}\right)^{-1}|r| .
$$

Further, we confirm that, under the above assumptions of Section 1 for the error functions $R_{i}$ and $r$ the following estimates hold:

$$
\begin{aligned}
& \|R\|_{\infty, \omega_{N}} \leq C N^{-1} \ln N, \\
& \left(\kappa_{0}\right)^{-1}|r| \leq C N^{-1} \ln N .
\end{aligned}
$$

The proof of (3.17) is almost identical to that in the work of Cakir and Amiraliyev [4]. We now estimate the remainder term $r$. From its explicit expression (3.9), under the smoothness conditions of Lemma 2.1, we get

$$
\begin{aligned}
\left(\kappa_{0}\right)^{-1}|r| & \leq C\left\{h_{1}+h_{3}+\int_{x_{0}}^{x_{1}}\left|u^{\prime}\right| d x+\int_{x_{N-1}}^{x_{N}}\left|u^{\prime}\right| d x\right\} \\
& \leq C\left\{h_{1}+h_{3}+\int_{x_{0}}^{x_{1}} \varepsilon^{-1} e^{-\frac{\mu_{1} x}{\varepsilon}} d x+\int_{x_{N-1}}^{x_{N}} \varepsilon^{-1} e^{-\frac{\mu_{2}(l-x)}{\varepsilon}} d x\right\} \\
& \leq C\left\{h_{1}+h_{3}+h_{1} / \varepsilon+h_{3} / \varepsilon\right\},
\end{aligned}
$$

which yields (3.18).

Now we can formulate the main convergence result:

Theorem 3.1. Let $u(x)$ be the solution of (1.1)-1.3) and $y$ the solution (3.10)-(3.12). Then the following estimate holds

$$
\|y-u\|_{\infty, \bar{\omega}_{N}} \leq C N^{-1} \ln N \text {. }
$$




\section{Algorithm and numerical results}

a) To solve the nonlinear problem $3.10-3.12$, we use the following quasilinearization technique:

$$
\begin{gathered}
\varepsilon^{2} \theta_{i} y_{\bar{x} \hat{x}, i}^{(n)}+\varepsilon a_{i} y_{x, i}^{(n)}-f\left(x_{i}, y_{i}^{(n-1)}\right)-\frac{\partial f}{\partial y}\left(x_{i}, y_{i}^{(n-1)}\right)\left(y_{i}^{(n)}-y_{i}^{(n-1)}\right)=0, \\
y_{0}^{(n)}-y_{N}^{(n)}=0 \\
\varepsilon^{2}\left(\theta_{0}^{(N)} y_{\bar{x}, N}^{(n)}-\theta_{0}^{(0)} y_{x, 0}^{(n)}\right)+\kappa_{0}\left(f\left(0, y_{0}^{(n-1)}\right)+\frac{\partial f}{\partial y}\left(0, y_{0}^{(n-1)}\right)\left(y_{0}^{(n)}-y_{0}^{(n-1)}\right)\right)=\varepsilon A, \\
n=1,2, \ldots ; \quad y_{i}^{(0)} \text { given, } \quad 1 \leq i \leq N-1 .
\end{gathered}
$$

b) Consider the test problem:

$$
\begin{aligned}
A & =1, \quad a(x)=1-\frac{x}{2}, \\
f(x, u) & =1+x^{2}+u+\tanh u, \quad 0<x<1 .
\end{aligned}
$$

For each value of $n$ the algorithm 4.1)-4.3 has been solved by the periodical factorization procedure (see [1, 2, 4 16]), with the initial guess $y_{i}^{(0)}=x_{i}-x_{i}^{2}$ and stopping criterion

$$
\max _{i}\left|y_{i}^{(n)}-y_{i}^{(n-1)}\right|<10^{-5} .
$$

As the exact solution is not known we use the double mesh technique to estimate the errors and the experimental rate of convergence in our computed solution. That is, we compare the computed solutions with the solution on a mesh that is twice as fine. The error estimate $e_{\varepsilon}^{N}$ and the computed convergence rate $P_{\varepsilon}^{N}$ obtained in this way are denoted by

$$
e_{\varepsilon}^{N}=\max _{\bar{\omega}_{N}}\left|y^{\varepsilon, N}-\tilde{y}^{\varepsilon, 2 N}\right|
$$

where $\tilde{y}^{\varepsilon, 2 N}$ is the approximate solution of the respective method on the mesh

$$
\tilde{\omega}_{2 N}=\left\{x_{i / 2}=0,1, \ldots .2 N\right\}
$$

with

$$
x_{i+1 / 2}=\left(x_{i}+x_{i+1}\right) / 2, \quad i=0, . ., N-1
$$

and

$$
P_{\varepsilon}^{N}=\ln \left(e_{\varepsilon}^{N} / e_{\varepsilon}^{2 N}\right) / \ln 2
$$

\section{Conclusion}

In this paper, the singularly perturbed periodical boundary value problem for a quasilinear secondorder differential equation is considered. We have constructed a numerical method for solving this problem, which generates $\varepsilon$-uniformly convergent numerical approximations to the solution and its derivatives. The method comprises a special non-uniform mesh, which is fitted to the boundary layers and constructed a priori in function of sizes of parameter $\varepsilon$ and the problem data. First order convergence in the discrete maximum norm, independently of the perturbation parameter is obtained. The exact errors and the rates of convergence are computed for different values of $\varepsilon$ and $N$ in Table 1 . The obtained results show that the convergence rate of difference scheme (3.10)-(3.12) is essentially in accord with the theoretical analysis. They indicate that the theoretical results are fairly sharp. The main lines for the analysis of the uniform convergence carried out here can be used for the study of more complicated differential problems with periodical as well as another type boundary conditions. 
Table 1: $\quad$ Errors $e_{\varepsilon}^{N}$ and convergence rates $P_{\varepsilon}^{N}$ for various values of $\varepsilon$ and $N$ on $\bar{\omega}_{N}$

\begin{tabular}{|c|c|c|c|c|}
\hline$\varepsilon$ & $N=32$ & $N=64$ & $N=128$ & $N=256$ \\
\hline $2^{-2}$ & 0.0115264 & 0.0068744 & 0.0036417 & 0.0017931 \\
& 0.80 & 0.88 & 0.94 & 0.99 \\
$2^{-4}$ & 0.0114367 & 0.0068732 & 0.0036410 & 0.0017630 \\
& 0.79 & 0.86 & 0.94 & 0.99 \\
$2^{-6}$ & 0.0114335 & 0.0068738 & 0.0036413 & 0.0017633 \\
& 0.79 & 0.86 & 0.94 & 0.99 \\
$2^{-8}$ & 0.0114346 & 0.0068735 & 0.0036415 & 0.0017632 \\
& 0.79 & 0.86 & 0.94 & 0.99 \\
$2^{-10}$ & 0.0114347 & 0.0068735 & 0.0036415 & 0.0017632 \\
& 0.79 & 0.86 & 0.94 & 0.99 \\
$2^{-12}$ & 0.0114345 & 0.0068735 & 0.0036415 & 0.0017632 \\
& 0.79 & 0.86 & 0.94 & 0.99 \\
$2^{-14}$ & 0.0114345 & 0.0068735 & 0.0036415 & 0.0017632 \\
& 0.79 & 0.86 & 0.94 & 0.99 \\
& & & & \\
$e^{N}$ & 0.0115264 & 0.0068744 & 0.0036417 & 0.0017931 \\
$P^{N}$ & 0.80 & 0.88 & 0.94 & 0.99 \\
\hline
\end{tabular}

\section{References}

[1] G. M. Amiraliyev, H. Duru, A uniformly convergent difference method for the periodical boundary value problem, Comput. Math. Appl., 46 (2003), 695-703. 1, 2, 3, 4

[2] G. M. Amiraliyev, Y. D. Mamedov, Difference schemes on the uniform mesh for singularly perturbed pseudoparabolic equations, Turkish J. Math., 19 (1995), 207-222. 1, 3, 4

[3] X. Cai, A conservative difference scheme for conservative differential equation with periodic boundary, Appl. Math. Mech. (English Ed.), 22 (2001), 1210-1215. 1

[4] M. Çakir, G. M. Amiraliyev, A numerical method for a singularly perturbed three-point boundary value problem, J. Appl. Math., 2010 (2010), 17 pages. 1, 3, 3, 4

[5] E. P. Doolan, J. J. H. Miller, W. H. A. Schilders, Uniform numerical methods for problems with initial and boundary layers, Boole Press, Dublin, (1980). 1

[6] P. A. Farrell, A. F. Hegarty, J. J. H. Miller, E. O'Riordan, G. I. Shishkin, Robust computational techniques for boundary layers, Chapman \& Hall/CRC, Boca Raton, (2000). 1

[7] J. L. Gracia, E. O'Riordan, M. L. Pickett, A parameter robust second order numerical method for a singularly perturbed two-parameter problem, Appl. Numer. Math., 56 (2006), 962-980. 1

[8] P. C. Lin, B. X. Jiang, A singular perturbation problem for periodic boundary differential equations (Chinese), Appl. Math. Mech., 8 (1987), 929-937. 1

[9] T. Linß, Layer-adapted meshes for reaction-convection-diffusion problems, Springer-Verlag, Berlin, (2010). 1

[10] T. Linß, H. G. Roos, Analysis of a finite-difference scheme for a singularly perturbed problem with two small parameter, J. Math. Anal. Appl., 289 (2004), 355-366. 1

[11] A. H. Nayfeh, Perturbation methods, Wiley-Interscience (John Wiley \& Sons), New York, (1973). 1

[12] R. E. O'Malley, Singular perturbation methods for ordinary differential equations, Springer-Verlag, New York, (1991). 1

[13] A. A. Pechenkina, Solution of the periodic problem for second order ordinary differential equation with small paramete in its leading derivatives, Ural Scientific Centre, Sverdlovsk, (1980), 111-117. 1

[14] J. I. Ramos, Exponentially-fitted methods on layer-adapted meshes, Appl. Math. Comput., 167 (2005), $1311-1330$. 1

[15] H. G. Roos, M. Stynes, L. Tobiska, Robust numerical methods for singularly perturbed differential equations, Springer-Verlag, Berlin, Heidelberg, (2008). 1

[16] A. A. Samarskii, Theory of difference schemes, Marcel Dekker, Inc., New York, (2001). 4

[17] G. Sun, M. Stynes, A uniformly convergent method for a singularly perturbed semilinear reaction diffusion problem with multible solutions, Math. Comput., 65 (1996), 1085-1109. 1 